\title{
Láser Er:YAG en Periodoncia. Revisión bibliográfica
}

\author{
HERRERO SANCHEZ A * \\ GARCIA NUÑEZ JA **
}

Herrero Sánchez A, García Núñez JA. Láser Er:YAG en Periodoncia. Revisión bibliográfica. Av Periodon Implantol. 2002; 14, 2: 63-68.

\begin{abstract}
RESUMEN
Diferentes láseres, como el $\mathrm{CO}_{2}$, Nd:YAG y ArF se han propuesto en la terapéutica periodontal. La mayor parte de ellos no han alcanzado relevancia clínica, debido a los resultados insatisfactorios obtenidos en las superficies radiculares tratadas, la generación de efectos colaterales o la ausencia de terminales de aplicación adecuados. Sin embargo, el nuevo láser Er:YAG se muestra como una prometedora alternativa en la remoción de placa bacteriana y cálculo de las superficies radiculares afectas, gracias a su mecanismo de ablación termomecánica y a la ausencia de efectos térmicos secundarios. El propósito de este artículo es revisar las actuales aplicaciones del láser Er:YAG en la práctica periodontal.
\end{abstract}

\section{PALABRAS CLAVE}

Superficie radicular, raspaje, cálculo, cemento, láser Er:YAG, láser/periodoncia, láser/efectos adversos.

\section{INTRODUCCIÓN}

Tras el desarrollo del láser de rubí por T. Maiman en 1960 , los láseres se han considerado como una alternativa diagnóstica y terapéutica en muchas ramas de la Medicina. En 1964, Stern y Sognnaes ${ }^{1}$ publicaron las primeras acciones de dicha tecnología en los tejidos duros dentarios. Desde entonces, muchos investigadores han estudiado las aplicaciones de los diferentes sistemas de láseres duros en Odontología, como la preparación de cavidades, la prevención de caries, la cirugía con láser, así como los efectos bioestimulantes, analgésicos y antiinflamatorios de los láseres blandos (tabla 1).

En los últimos diez años, se han publicado numerosos estudios con relación a las aplicaciones del láser en Periodoncia. En este sentido, se han empleado los láseres de $\mathrm{CO}_{2}$, Nd:YAG, He-Ne, ArGa, de excímeros y, últimamente el Er:YAG en el tratamiento de las superficies radiculares y en la cirugía de tejidos blandos. Sin embargo, muchos de ellos no han logrado un desarrollo importante, debido a las escasas ventajas, y en algunos casos inconvenientes, que presentan en relación con los medios tradicionales de tratamiento ${ }^{2}$. Además, la mayor parte de los estudios publicados carecen de evidencia científica contrastada de sus acciones.

El método convencional de tratamiento de los tejidos periodontales es el raspado y alisado, con o sin acceso quirúrgico, empleando métodos mecánicos manuales como las curetas, o aparatos sónicos y ultrasónicos ${ }^{3}$. La finalidad de dicha terapéutica es la reducción de las bolsas periodontales, mediante la remoción de placa bacteriana, cálculo y toxinas presentes en la superficie radicular, creando así una superficie biológicamente compatible que evite la progresión o recurrencia de la enfermedad ${ }^{4}$.

Diversos estudios indican que la anatomía radicular, la

* Doctorando en Odontología. Facultad de Odontología. Universidad Complutense de Madrid.

** Juan Antonio García Núñez. Prof. Titular de Medicina Bucal y Periodoncia. Facultad de Odontología. Universidad Complutense de Madrid.

Departamento de Medicina y Cirugía Bucofacial. Facultad de Odontología. Universidad Complutense de Madrid. 


\begin{tabular}{|c|c|c|c|c|c|c|}
\hline TIPO DE LÁSER & $\begin{array}{l}\text { MEDIO } \\
\text { ACTIVO }\end{array}$ & $\begin{array}{l}\text { LONGITUD } \\
\text { DE ONDA }(\mu \mathrm{m})\end{array}$ & EMISIÓN & $\begin{array}{l}\text { POTENCIA } \\
\text { (W) }\end{array}$ & $\begin{array}{c}\text { HAZ } \\
\text { PILOTO }\end{array}$ & EFECTOS \\
\hline \multicolumn{7}{|l|}{$\begin{array}{l}\text { - Láser blando } 0 \\
\text { terapéutico }\end{array}$} \\
\hline 1. $\mathrm{He}-\mathrm{Ne} \mathrm{Ga}$ & Gaseoso & $\begin{array}{l}\text { Visible } \\
0,63 \mu \mathrm{m} \text { (rojo) } \\
0,54 \mu \mathrm{m} \text { (verde) }\end{array}$ & Continua & $2-50 \mathrm{~mW}$ & No & $\begin{array}{l}\text { Bioestimulante } \\
\text { Analgésico } \\
\text { Antiinflamatorio }\end{array}$ \\
\hline 2. ArGa (diodo) & Sólido & $\begin{array}{l}\text { Invisible } \\
0,9 \mu \mathrm{m} \\
\text { (infrarrojo) }\end{array}$ & Continua & $3-20 \mathrm{~W}$ & $\mathrm{Si}$ & $\begin{array}{l}\text { Bioestimulante } \\
\text { Analgésico } \\
\text { Antiinflamatorio }\end{array}$ \\
\hline \multicolumn{7}{|l|}{$\begin{array}{l}\text { - Láser duro o } \\
\text { quirúrgico }\end{array}$} \\
\hline 1. Argón & Gaseoso & $\begin{array}{l}\text { Visible } \\
0,48 \mu \mathrm{m} \text { (azul) } \\
0,54 \mu \mathrm{m} \text { (verde) }\end{array}$ & Continua & $0,5-1000 \mathrm{~W}$ & No & $\begin{array}{l}\text { Fotocoagulador } \\
\text { Fotopolimerizador de RC }\end{array}$ \\
\hline 2. Nd:YAG & Sólido & $\begin{array}{l}\text { Invisible } \\
1,06 \mu \mathrm{m} \\
\text { (infrarrojo) }\end{array}$ & $\begin{array}{l}\text { Pulsante } \\
\text { Continua }\end{array}$ & $20-120 \mathrm{~W}$ & Si & $\begin{array}{l}\text { Efecto térmico } \\
\text { Corte } \\
\text { Coagulación }\end{array}$ \\
\hline 3. $\mathrm{CO} 2$ & Gaseoso & $\begin{array}{l}\text { Invisible } \\
10,6 \mu \mathrm{m} \text { (infrarrojo } \\
\text { lejano) }\end{array}$ & $\begin{array}{l}\text { Pulsante } \\
\text { Continua }\end{array}$ & $0,5-1000 \mathrm{~W}$ & Sí & $\begin{array}{l}\text { Efecto térmico importante } \\
\text { Corte } \\
\text { Coagulación }\end{array}$ \\
\hline $\begin{array}{l}\text { 4.Excimero } \\
\text { (ArF,XeCl) }\end{array}$ & Gaseoso & $\begin{array}{l}\text { Invisible } \\
0,15.0,35 \mu \mathrm{m} \\
\text { (ultraviole-ta) }\end{array}$ & Pulsante & $100 \mathrm{~W}$ & $\mathrm{Si}$ & Corte \\
\hline 5.Er:YAG & Sólido & $\begin{array}{l}\text { Invisible } \\
2,94 \mu \mathrm{m}\end{array}$ & Pulsante & $50-500 \mathrm{~mJ}$ & $\mathrm{Si}$ & $\begin{array}{l}\text { Corte } \\
\text { Ablación termomecánica } \\
\text { Escasa coagulación }\end{array}$ \\
\hline
\end{tabular}

Tabla 1

morfología y profundidad de la bolsa periodontal o una técnica inadecuada limitan estos procedimientos. Así, tras la instrumentación con curetas se observa cálculo residual y una capa de barrillo dentinario o smear layer, constituida por bacterias y mediadores citotóxicos. Esta película podría dificultar la reinserción de los fibroblastos y en consecuencia, retardar o impedir la curación del proceso ${ }^{5}$.

Existen diferentes opiniones con relación a los ultrasonidos, aunque la mayor parte de los estudios avalan su eficacia ${ }^{6.7}$.

En los últimos años han aparecido nuevos diseños de curetas y ultrasonidos, así como ácidos y polvos abrasivos, presentando igualmente limitaciones ${ }^{8,9}$. Así, el ácido cítrico, empleado en el acondicionamiento de las superficies radiculares, genera un ensanchamiento de los túbulos dentinarios ${ }^{10}$. Los abrasivos pulverizados facilitan la detoxificación en aquellas zonas donde los instrumentos mecánicos no acceden; sin embargo, traumatizan los tejidos blandos, desmineralizan las superficies dentarias y generan sensibilidad ${ }^{11}$.

En la búsqueda de terapéuticas eficaces para lograr una superficie radicular biocompatible, aparecen los láseres en el campo de la Periodoncia. En un principio, se emplearon los láseres de $\mathrm{CO}_{2}$ y $\mathrm{Nd}$ :YAG, dando 
lugar a importantes daños térmicos, debido a las altas densidades energéticas requeridas para la instrumentación ${ }^{2}$

Algunos autores, como Ito y cols. ${ }^{12}$, expusieron que el láser de Nd:YAG podría ser empleado en la eliminación del smear layer generado tras la instrumentación con curetas. Según ellos, que se produce una remoción eficaz de dicha película, con exposición de los túbulos dentinarios, pero sin ensanchamiento, a diferencia de lo observado con el ácido cítrico.

Sin embargo, como exponen Tseng y cols. ${ }^{13}$, el láser de Nd:YAG no consigue eliminar de forma significativa el cálculo subgingival, sólo lo desprende ligeramente del cemento. Por lo tanto, sería necesario realizar posteriormente una instrumentación con curetas.

Resultados semejantes muestran los estudios de Misra y cols. ${ }^{14}$ con el láser de $\mathrm{CO}_{2}$. Según ellos, este láser es capaz de eliminar el barrillo dentinario con menor ensanchamiento de los túbulos dentinarios que el tratamiento con $\mathrm{H}_{2} \mathrm{O}_{2}$, EDTA o ácido cítrico.

Las imágenes observadas por los diferentes autores muestran alteraciones morfológicas importantes en las superficies radiculares tratadas con láser de $\mathrm{CO}_{2}$ y Nd:YAG. Así, se han descrito fracturas microscópicas, cráteres, carbonización, fusión y recristalización de la fase mineral. Se advierten zonas de ablación atenuadas y exposición del cemento, incluso a energías bajas ${ }^{12,14}$.

El láser de Er:YAG se muestra como una posible alternativa a los medios mecánicos actuales en el tratamiento de las superficies radiculares. El mecanismo de acción de este láser, denominado ablación termomecánica, se basa en la alta absorción de su radiación $(2,94 \mu \mathrm{m})$ por el agua superficial y por los grupos $\mathrm{OH}-$ de la hidroxiapatita. La elevación de la temperatura del agua da lugar a la evaporación de la misma, aumentado la presión intratisular. En consecuencia, se generan microexplosiones con la consiguiente ablación del tejido. Al incidir únicamente a nivel superficial, no se generan daños térmicos subyacentes ${ }^{15}$.

Sin embargo, los primeros terminales impedían una adecuada instrumentación en el interior de las bolsas periodontales. Gracias a diferentes investigaciones, se diseñó una fibra de cuarzo con punta biselada para facilitar su aplicación ${ }^{16}$.

Los diferentes autores consultados proponen rangos energéticos muy dispares en la instrumentación radicular con láser de Er:YAG. Según Stock y cols. ${ }^{17}$, esto puede deberse al diseño experimental, a la técnica de aplicación, así como a variables cualitativas y cuantitativas del cálculo (color, textura, contenido en agua o espesor).
En este sentido, Aoki y cols. ${ }^{18}$ realizaron un estudio "in vitro" aplicando el láser Er:YAG con rangos energéticos crecientes. Exponen que el proceso de ablación del cálculo subgingival se acompaña de desprendimiento de cemento y dentina, a menos que las densidades energéticas empleadas sean inferiores a 10,6 $\mathrm{J} / \mathrm{cm}^{2} /$ pulso (30 mJ/pulso), para las cuales se elimina únicamente cálculo.

Stock y cols. ${ }^{17}$ exponen que para la radiación del láser Er:YAG, el cálculo y el cemento radicular presentan el mismo umbral de ablación, situado a $13 \mathrm{~J} / \mathrm{cm}^{2} /$ pulso (37 $\mathrm{mJ} /$ pulso); por tanto, no es posible la remoción selectiva del cálculo radicular. Al igual que Aoki y cols ${ }^{18}$, indican que la ablación completa del tártaro subgingival siempre conllevará eliminación de cemento.

Keller y Hibst ${ }^{19}$ en investigaciones anteriores, muestran que densidades energéticas de $17,5 \mathrm{~J} / \mathrm{cm}^{2} / \mathrm{pulso}$ (50 mJ/pulso) no ocasionan daños en el cemento radicular. Indican que la ablación eficaz del cálculo y cemento infectado se logra con $21-52,5 \mathrm{~J} / \mathrm{cm}^{2} /$ pulso (60-150 mJ/pulso).

La morfología radicular obtenida tras la instrumentación con láser Er:YAG también ha sido motivo de estudio por diferentes autores. Así, Aoki y cols. ${ }^{18}$ indican que las superficies radiculares tratadas muestran un aspecto irregular, tizoso y con cráteres asilados. Según Fujii y cols. ${ }^{20}$, dichos cráteres podrían favorecer la recolonización bacteriana.

Folwaczny y cols. ${ }^{16}$ indican que energías superiores a $35 \mathrm{~J} / \mathrm{cm}^{2} /$ pulso (100 mJ/pulso) eliminan el cálculo radicular sin formación de barrillo o smear layer. Según ellos, las imágenes son similares a las mostradas por las curetas y ultrasonidos. Estos autores, al igual que Aoki y cols. ${ }^{18}$ no observan signos de carbonización y fusión. Sin embargo, en un estudio posterior efectuado por Aoki y cols. ${ }^{21}$ se evidencia que la eliminación del cálculo con láser Er:YAG es menor que con ultrasonidos.

Keller y Hibst ${ }^{19}$ describen una superficie irregular, con huellas de ablación de bordes romos y profundidad máxima de $100 \mu \mathrm{m}$ causadas por el desplazamiento de la fibra óptica. Indican que el cemento muestra una imagen homogénea, con impactos de láser aislados en forma de cráter de fondo irregular y $270 \mu \mathrm{m}$ de profundidad. En estudios posteriores exponen que existe relación entre la morfología de la superficie radicular tratada y la inclinación de la fibra óptica. Así, proponen situarla en un ángulo de $20^{\circ}$, aunque se precisen energías mayores de ablación, porque dicha angulación disminuye la probabilidad de daño térmico ${ }^{22}$.

Según los autores consultados, las superficies tratadas 
con láser Er:YAG no muestran carbonización, a diferencia de las instrumentadas con láser $\mathrm{CO}_{2}$ o Nd:YAG ${ }^{16,17,19}$. Esto parece tener implicaciones clínicas importantes. Así, estudios "in vivo" e "in vitro" indican que la presencia de una película carbonizada residual en las superficies radiculares inhibe la inserción fibroblástica ${ }^{11}$.

En este sentido, se ha propuesto que el calentamiento de la hidroxiapatita, en presencia del ión amonio, procedente de la degradación del colágeno, da lugar a la aparición de iones cianamida y cianato. Según Thomas ${ }^{11}$, estos iones son tóxicos para el fibroblasto, lo cual podría confirmar las observaciones realizadas por Aberguel ${ }^{23}$ en fibroblastos de piel irradiados con láser Nd:YAG. Según este autor, se produce un retardo en la síntesis de colágeno y DNA.

También ha sido objeto de estudio por varios autores la inserción de los fibroblastos a las superficies radiculares tratadas con láser Er:YAG. Aunque existe cierta controversia para determinar si se consigue una mejor inserción fibroblástica en superficies lisas o rugosas ${ }^{24}$, Folwaczny y cols. ${ }^{16}$ indican que la superficie tratada con Er:YAG es semejante a la observada por el tratamiento con EDTA, lo cual para algunos autores, mejora el resultado del tratamiento ${ }^{25}$. Estos autores, así como Stock y cols. ${ }^{17}$ sugieren que la superficie irregular obtenida tras el tratamiento con Er:YAG podría favorecer la inserción de los fibroblastos.

Una de las líneas de investigación en este campo se orienta a determinar el poder bactericida de los láseres. Así, Cobb y cols. ${ }^{26}$ empleando un láser de Nd:YAG y sondas de DNA, advirtieron una disminución en los niveles de Actinobacillus actinomycetemcomitans, Porphyromonas gingivalis, y Prevotella intermedia. A los siete días de la irradiación, observaron al microscopio electrónico de barrido la recolonización bacteriana. Kato y cols. ${ }^{27}$ observan el poder bactericida in vitro del láser $\mathrm{CO}_{2}$ para Porphyromonas gingivalis.

Ando y cols. ${ }^{28}$ exponen que el efecto bactericida del láser Er:YAG se basa en la alta absorción de esta radiación por el agua intracelular, que dará lugar a una evaporación de la misma y explosión de la bacteria; además, según ellos, el calor generado por la radiación producirá un efecto letal añadido para los microorganismos. Fundamentándose en estos principios, realizaron un experimento "in vitro" a fin de estudiar el poder antibacteriano del láser para Porphyromonas gingivalis y Actinobacillus actinomycetemcomitans. Observaron que energías de 0,3-0,4 $\mathrm{J} / \mathrm{cm}^{2}$, inhiben el crecimiento de estas bacterias.

Yamaguchi y cols. ${ }^{29}$ estudiaron el efecto del láser Er:YAG en los lipopolisacáridos (LPS) de las bacterias gram -. Según ellos, los LPS presentan un pico de absorción alto para la longitud de onda de 2,94 $\mu \mathrm{m}$. Observan que el láser elimina el $83,1 \%$ de los LPS, bien por el efecto del calor o por la ablación de los tejidos en los que se encuentran.

El láser Er:YAG también ha sido empleado en cirugía periodontal. Así, Keller y Hibst ${ }^{30}$ exponen la eficacia de este láser en gingivectomías, gingivoplastias, frenectomías. Indican que las incisiones realizadas muestran bordes bien definidos y con reducidas zonas de necrosis, lo que favorecerá la cicatrización de las heridas. Según ellos, el tiempo de curación es similar al observado con bisturí convencional. Sin embargo, debido al escaso poder hemostático de este láser, recomiendan no emplearlo en lesiones muy inflamadas.

Watanabe y cols. ${ }^{31}$ realizaron un estudio en humanos aplicando este láser en gingivectomías y frenectomías. Según ellos, cuando se emplea sin terminal, se reduce el sangrado intraoperatorio al coagular los vasos sanguíneos y al generarse una capa de degeneración proteica. Sin embargo, indican que el efecto hemostático no es excesivamente alto si se compara con el láser de $\mathrm{CO}_{2}$ y de $\mathrm{Nd}$ :YAG.

Estos autores exponen que a los siete días de realizar la gingivectomía se reduce la profundidad de bolsa; a las dos semanas, la inflamación y el enrojecimiento disminuyen significativamente. Indican que la reepitelización de la herida se produce a la semana y la curación total a los treinta días.

Verdasco y Ortiz ${ }^{15}$ aconsejan emplear el láser Er:YAG en los tejidos blandos con energías bajas y frecuencias altas para realizar cortes lineales, pudiéndose aplicar sin irrigación para obtener cierta hemostasia en los bordes, aunque en las cercanías del periostio se debe emplear siempre con refrigeración. Indican la importancia de la aspiración quirúrgica, para no disminuir la eficacia de corte de este láser.

También se han empleado los láseres de $\mathrm{CO}_{2}, \mathrm{Nd}$ :YAG y de Diodos en la cirugía periodontal. En este sentido, ciertos autores muestran resultados eficaces con el láser $\mathrm{CO}_{2}$ en gingivectomías de hiperplasias gingivales, especialmente las causadas por fenitoina ${ }^{32}$ y nifedipina ${ }^{33}$. Según ellos, este láser permite un campo exangüe, por su efecto hemostático, y estéril, reduciendo así el riesgo de bacteriemia; esto puede ser beneficioso en pacientes inmunocomprometidos.

\section{CONCLUSIONES}

1. Las altas temperaturas generadas por los láseres de $\mathrm{CO}_{2}$ y de Nd:YAG los invalida en el tratamiento de 
los tejidos duros dentarios, siendo el láser de Er:YAG una posible alternativa terapéutica a los medios mecánicos actuales en la instrumentación periodontal.

2. La mayor parte de los estudios exponen que el láser de Er:YAG es eficaz para eliminar el cálculo subgingival, siempre y cuando se aplique de forma adecuada.

3. En la cirugía de los tejidos blandos periodontales, los láseres más empleados son el de $\mathrm{Nd}$ :YAG y $\mathrm{CO}_{2}$ debido a su alto poder hemostático y de corte.

4. Se ha descrito el efecto bactericida "in vitro" del láser Er:YAG para Porphyromonas gingivalis y Actinobacillus actinomycetemcomitans.

\section{ABSTRACT}

Different lasers, such as $\mathrm{CO}_{2}$, the Nd:YAG and the ArF have been proposed for use in periodontal therapy. Most systems have not reached clinical significance because of the unsattisfactory results of laser treated root surfaces, emergence of severe side effects, or the lack of suitable delivery systems. However, the recently introduced Er:YAG laser radiation appears to be a promising alternative means of removing microbial plaque and calculus from diseased tooth root surfaces, due to its thermo-mechanical ablation properties and the lack of thermal side effects. The purpose of this article is to review current applications of laser Er:YAG to periodontal practice.

\section{KEY WORDS}

Tooth root, scaling, dental calculus, cementum, Er:YAG laser, lasers/periodontic, lasers/adverse effects.

\section{CORRESPONDENCIA}

J.A. García Núñez

Facultad de Odontología

P/ Ramón y Cajal s/n

Ciudad Universitaria

28040 Madrid. España

E-mail:garcinu@eucmos.sim.ucm.es

\section{BIBLIOGRAFÍA}

1. Stern RH, Sognnaes RF. Laser beam effect on hard tissues. J S Calif Dent Ass 1964; 23: 17-9.

2. Cobb C. Lasers in periodontics: use and abuse. Compend 1997; 18: 847-59.

3. Sbordone L, Ramaglia L, Gulletta E, Iancono V. Recolonization of the subgingival microflora after scaling and root planing in human periodontitis. J Periodontol 1990; 61: 579-84.

4. Buchanan SA, Robertson PB. Calculus removal by scaling root planing with and without surgical access. J Periodontol 1987; 58: 159-63.

5. Stambaugh RV, Dragoo M, Smith DM, Carasali L. The limits of subgingival scaling. Int J Periodont Rest Dent $1981 ; 1: 31-41$.

6. Breininger DR, O'Leary TJ, Blumenshire RVH. Comparative effectiveness of ultrasonic and hand scaling for the removal of subgingival plaque and calculus. J Periodontol 1987; 58: 9-18.

7. García JA, Ramos JM, Cerero R, Esparza GC. Resultados de las técnicas de profilaxis a la luz de la M.E.B. Av Odontoestomatol 1984; 2: 83-6.

8. Berkstein S, Reiff RL, McKinney JF, Killoy WJ. Supragingival root surface removal during maintenace procedures utilizing an air power abrasive system or hand scaling. J Periodontol 1987; 58: 327-9.

9. Gankerseer E, Walmsley A. Preliminary investigation into the performance of a sonic scaler. J Periodontol 1987; 58: 780-4.

10. Lasho DJ, O'Leary TJ, Kafrawy AH. A scanning electron microscopic study of the effects of varius agents on instrumented periodontally involved root surfaces. J Periodontol 1983; 54: 210-20.

11. Thomas D, Rapley J, Cobb C, Spencer P, Killoy W. Effects of the Nd:YAG laser and combined treatments on in vitro fibroblast attachment to root surfaces. J Clin Periodontol 1994; $21: 38-44$.

12. Ito K, Nishikata J, Murai S. Effect of Nd:YAG laser radiation on removal of a root surface smear layer after root planing: a scanning electron microscopic study. J Periodontol 1993; 64: 547-52.

13. Tseng P, Gilkeson CF, Palmer J, Liew V. The bactericidal effect of a Nd:YAG laser in vitro (abstract 7). J Dent Res 1991; 70: 650 . 
14. Misra V, Mehrotra KK, Dixit J, Maitra SC. Effect of a carbon dioxide laser on periodontally involved root surfaces. J Periodontol 1999; 70: 1046-52.

15. Verdasco M, Ortiz B. Láser erbio-YAG: principios físicos y aplicaciones en odontología. Quintessence (ed. Esp) 1996; 9: 657-68.

16. Folwaczny M, Mehl A, Haffner C, Benz C, Hickel R. Root substance removal with Er:YAG laser radiation at different parameters using a new delivery system. J Periodontol 2000; 71: 147-55.

17. Stock K, Hibst R, Keller U. Er:YAG removal of subgingival calculus: efficiency, temperature and surface quality. Proc SPIE 1996; 2922: 98-105..

18. Aoki A, Ando Y, Watanabe H, Ishikawa I. In vitro studies on laser scaling of subgingival calculus with an Erbium:YAG laser. J Periodontol 1994; 65: 1097-106.

19. Keller U, Hibst R. Experimental removal of subgingival calculus with the Er:YAG laser. Proc SPIE 1995; 2623: 18998.

20. Fujii T, Baehni PC, Kawai O, Kawakami T, Matsuda K, Kowashi Y. Scanning electron microscopic study of the effects of Er:YAG laser on root cementum. J Periodontol 1998; 69: 1283-90.

21. Aoki A, Miura M, Akiyama F, Nakagawa N, Tanaka J, Oda $\mathrm{S}$, Watanabe $\mathrm{H}$, Ishikawa I. In vitro evaluation of Er:YAG laser scaling of subgingival calculus in comparison with ultrasonic scaling. J Periodont Res 2000; 35: 266-77.

22. Keller U, Stock K, Hibst R. Morphology of Er:YAG lasertreated root surfaces. Proc SPIE 1997; 3192: 24-31.

23. Aberguel RP, Meeker CA, Dwyer RM, Lesavoy MA, Vitto J. Non-thermal effects of Nd:YAG laser on biological functions of human skin fibroblast in culture. Lasers Surg Med 1984; 3: 279-84.
24. Khatiblou FA, Ghodssi A. Root surface smoothness or roughness in periodontal treatment. J Periodontol 1983; 54: 365-7.

25. Albair WB, Cobb CM, Killoy WJ. Connective tissue attachment to periodontally diseased roots after citric acid demineralization. J Periodontol 1982; 53: 515-26.

26. Cobb CM, McCawley TK, Killoy WJ. A preliminary study on the effects of the Nd:YAG laser on root surfaces and subgingival microflora in vivo. J Periodontol 1992; 63: 701-7.

27. Kato T, Kusakari H, Hoshino E. Bactericidal efficacy of carbon dioxide laser against bacteria contaminated titanium implant and subsequent cellular adhesion to irradiated area. Lasers Surg Med 1998; 23: 299-309.

28. Ando Y, Aoki A, Watanabe H, Ishikawa I. Bactericidal effect of Erbium:YAG laser. Lasers Surg Med 1996; 19: 190-200.

29. Yamaguchi H, Kobayashi K, Osada R, Sakuraba E, Nomura T, Arai T, Nakamura J. Effects of irradiation of an Erbium:YAG laser on root surfaces. J Periodontol 1997; 68: 1151-5.

30. Keller U, Hibst R. Lasers in oral surgery. Medical applications of lasers II. Proc SPIE 1994; 2327: 146-54.

31. Watanabe H, Ishikawa I, Suzuki M, Hasegawa K. Clinical assesments of the Erbium:YAG laser for soft tissue surgery and scaling. Lasers Surg Med 1996; 14: 67-75.

32. Pick RM, Pecaro BC, Silberman CJ. The laser gingivectomy. The use of the CO2 laser for the removal of phenytoin hiperplasia. J Periodontol 1985; 56: 492-6.

33. Barak S, Kaplan I. The CO2 laser in the excision of gingival hyperplasia caused by nifedipine. J Clin Periodontol 1988; 15: 633-5. 